\title{
Shell-model calculations for upper pf-shell nuclei with an effective interaction
}

\author{
Kalin Drumev ${ }^{1, *}$ \\ ${ }^{1}$ Institute for Nuclear Research and Nuclear Energy, Bulgarian Academy of Sciences, Sofia 1784, Bulgaria
}

\begin{abstract}
Results obtained for the energy spectra and the low-lying positive-parity energy eigenstates of the upper $p f$-shell nuclei ${ }^{64} \mathrm{Ge}$ and ${ }^{68} \mathrm{Se}$ with the use of the effective interaction JUN45 are reported. We address the question of how appropriate is the possibility to construct a symmetry-adapted shell model in a single oscillator shell using a Pairing-plus-Quadrupole Hamiltonian. Specifically, we study the goodness of the symmetries pseudo $S U(3)$ and $O(6)$ in the structure of the energy eigenstates. Finally, we relate our results to a proposed mixed-symmetry approach which is able to simultaneously account for the presence of both the pairing and the quadrupole modes as the most important ingredients in the effective interaction while using a restricted part of the full model space.
\end{abstract}

\section{Introduction}

Due to the enormous complexity of the nuclear systems and the lack of the complete and exhaustive knowledge of the strong nucleon-nucleon potential, there are variety of methods and models developed during the years for the successful description of various features of the atomic nuclei. Some of their properties (like the energy spectra, radii, etc.) can be easily described with the use of just a single shell within the shell-model approach. Others (like the building of collectivity and the correct description of electromagnetic transition strengths without the use of an effective charge) require from the same type of approach an expansion of the model space to a one for multishell calculations. Such an action is extremely challenging computationally. Thus, some important questions arise. For example, can we confirm that a certain symmetry (either exact, or most likely, dynamical) is present in the system? Then, we are tempted to also check whether such a fact can be revealed by using some realistic nucleonnucleon interaction, i.e. one which is consistent with data obtained in two- (and nowadays three-) nucleon systems.

Furthermore, there are cases when no one among several competing dynamical symmetries dominates. Still, it is worth testing the interplay between these modes. Sometimes, it is useful to introduce a mixed-mode approach in which these several competing modes can be put together and as a result to facilitate the calculations. It became quite natural during the years to represent different dynamical symmetries on symmetry diagrams (like in the Interacting Boson Model [1], where three possible dynamical symmetries - $U(5)$ - for a spherical vibrator, $O(6)$ - for a gammasoft rotor and the $S U(3)$ - for a rigid rotor - all in the $s d$ shell nuclei represent the vertices on a symmetry triangle).

In this work, we evaluate the goodness of the pseudo$S U(3)$ and $O(6)$ dynamical symmetries in the energy

\footnotetext{
*e-mail: kdrumev2000@yahoo.com
}

eigenfunctions. For the purpose, we calculate the overlaps of the corresponding eigenstates with the low-lying energy eigenstates in the $p f$-shell nuclei ${ }^{64} \mathrm{Ge}$ and ${ }^{68} \mathrm{Se}$. For these unrestricted shell-model calculations, we use the effective interaction JUN45, which was applied for the description of variety of properties in the these two and other upper $p f$-shell nuclei. Our results are quite promising for the development of a symmetry-adapted shell model which can combine the use of several dynamical symmetries while restricted number of basis states are needed for the successful achievement of a number of important results.

\section{The $\mathrm{N}=\mathrm{Z}$ nuclei ${ }^{64} \mathrm{Ge}$ and ${ }^{68} \mathrm{Se}$}

The $N \sim Z$ nuclei are an important area of the nuclear chart to be studied. This region is the arena of an interesting competition between the pairing and the quadrupolequadrupole interactions as the most important ingredients of the short- and long-range nucleon-nucleon correlations. The first is responsible for the existence of the energy gap in some nuclei near the closed shell while the latter explains the strong electromagnetic transition strengths. In the nuclear chart, these nuclei lie on the path of the rapid proton capture process (the rp-process) of nucleosynthesis. Furthermore, for the odd-odd isotopes, it is extremely interesting to study the role of the isoscalar pairing. Finally, these nuclei are a computational challenge since full-space shell model calculations, especially in a model space that includes more than a single oscillator shell, are often beyond our current computational capabilities. Therefore, finding good dynamical symmetries can be of great help.

As $N \sim Z$ nuclei from the upper $p f$ shell, the isotopes ${ }^{64} \mathrm{Ge}$ and ${ }^{68} \mathrm{Se}$ are special in some of the ways mentioned above. They both are known to be waiting-point nuclei on the path of the rp-process of nucleosynthesis, so the detailed knowledge of their energy eigenfunctions will be 
extremely valuable for the correct description of the rates of the nuclear reactions involved.

The fact that the nucleus ${ }^{64} \mathrm{Ge}$ behaves as a gamma soft rotor has been proposed by various theoretical investigations. The calculated excitation energy ratio $E_{x}\left(4_{1}^{+}\right) / E_{x}\left(2_{1}^{+}\right)=2.50$ from the experiment is in a reasonable agreement with the experimental value 2.27 which corresponds to a gamma-soft nucleus according to the Wilets-Jean model [2]. On the other hand, according to the rigid rotor model with triaxial deformation [3], the calculated ration between the strengths $B\left(E 2 ; 2_{2}^{+} \rightarrow\right.$ $\left.2_{1}^{+}\right) / B\left(E 2 ; 2_{2}^{+} \rightarrow 0_{1}^{+}\right)=14$ corresponds to a value for the deformation parameter of $\gamma \sim 23$ degrees - a result that suggests triaxiality for this isotope. In short, because of the reasons stated above, we expect this nucleus to behave as a gamma-soft rotor which case the Interacting Boson Model associates with the $O(6)$ dynamical symmetry.

On the hand, the isotope ${ }^{68} \mathrm{Se}$ is a nucleus where the effects of shape coexistence have been observed [4]. This system was extensively studied using different methods and approaches including large scale shell-model calculations. As we will see below, if calculations for this isotope are done in the upper $p f$-shell model space then the two leading representations of the $S U(3)$ algebra with $S U(3)$ labels $(\lambda, \mu)=(12,0)$ and $(0,12)$ suggest for the states from the ground and the excited band a similar quadrupole deformation but of the opposite sign - one of them corresponds to a prolate, the other - to an oblate shape.

\section{The shell-model approach for the upper $p f$-shell nuclei}

The shell model approach (from its quite naive formulation as a independent-particle model to the much more sophisticated interacting shell model using number of effective interactions of two- and even three-body nature) has been quite successful during the years for the description of various properties of the atomic nuclei along the whole nuclear chart. A review with the progress and the recent status can be found in [5]. Alternatively, people have also used phenomenological type of interactions like a Hamiltonian of the harmonic oscillator term plus other singleparticle pieces and the pairing plus quadrupole-quadrupole part as a residual interaction introduced to account for the short and long-range part correlations between the nucleons and to implement the configuration mixing.

In many cases, the matrices that need to be evaluated in the conventional shell model are huge - on the order of hundreds of millions and even tens of billions basis states. This is why one frequently asks the question: can one do these calculations more economically, which means in a restricted model space based on the existence of a certain single dynamical symmetry. Or, if not, can one use a mix of several dynamical symmetries whose interplay may be realized in a subspace of the whole model space thus allowing an easier calculation procedure?

\section{Projection method and renormalization procedure for the effective interaction}

One of our goals is to estimate the quality of some dynamical symmetries in the two upper $p f$-shell shell. Thus, part of our work is to make a transformation from the full model space $p f_{5 / 2} g_{9 / 2}$ to the restricted one $\left(p f_{5 / 2}\right)$ where the quality of this symmetry will be evaluated. To go to a subspace of the initial model space, we can use the projection method and follow the procedure described in $[6,7]$ to project the interaction by reducing the model space where it acts. A characteristic feature of this method is that it exactly reproduces the energy spectrum in the bigger model space before the renormalization was applied.

Next, we briefly explain how this method works. Let us consider a system of $n$ identical nucleons distributed among the single-particle shell-model levels of a given valence space. The basis states of this $n$-particle system, designated as $|I\rangle,|J\rangle$, define the full space $D$. The Hamiltonian $H$ in this full space consists of a one-body term $H_{0}$ and a two-body interaction $V$. In the full space $D$ we have in obvious notations $H\left|\psi^{\alpha}\right\rangle \equiv\left(H_{0}+V\right)\left|\psi^{\alpha}\right\rangle=E^{\alpha}\left|\psi^{\alpha}\right\rangle$, $H_{0}|I\rangle=E_{I}|I\rangle$ and $\left|\psi^{\alpha}\right\rangle=\sum_{I} c_{I}^{\alpha}|I\rangle$. Then, we consider a truncated space $d$ lying in $D$. The $n$-particle basis states in $d$ are denoted by $|i\rangle,|j\rangle$ while the symbols $\mathcal{I}, \mathcal{J}$ are reserved to the basis states in the omitted (i.e. not included in $d$ ) space. We want to construct an effective Hamiltonian $H_{e f f}$ in $d$. In the projection method $H_{e f f}$ is required to satisfy $H_{e f f}\left|\phi^{\alpha}\right\rangle=E^{\alpha}\left|\phi^{\alpha}\right\rangle$ with $\left|\phi^{\alpha}\right\rangle=\hat{P}\left|\psi^{\alpha}\right\rangle . E^{\alpha}$ and $\psi^{\alpha}$ are a subset (to be conveniently chosen) of the exact eigenvalues and of the corresponding eigenvectors; the operator $\hat{P}$ projects onto the model space $d$. Defining an operator $\hat{Q}$, which projects outside $d$, one has $\left|\phi^{\alpha}\right\rangle=(\hat{P}+\hat{Q})\left|\psi^{\alpha}\right\rangle=$ $\sum_{i \in d} c_{i}^{\alpha}|i\rangle+\sum_{\mathcal{I} \in D} c_{\mathcal{I}}^{\alpha}|\mathcal{I}\rangle$.

In one of the formulations of the method one can start from the normalized projections $\left|\phi_{\text {norm }}^{\alpha}\right\rangle=\left|\phi^{\alpha}\right\rangle /\left\langle\phi^{\alpha} \mid \phi^{\alpha}\right\rangle^{1 / 2}$ and defines the set of biorthogonal states $\left|\tilde{\phi}^{\alpha}\right\rangle$ such that $\left\langle\tilde{\phi}^{\beta} \mid \phi^{\alpha}\right\rangle=\delta_{\alpha \beta}$. To determine the components of the $\left|\tilde{\phi}^{\alpha}\right\rangle$ one has to invert the matrix of the $\left|\phi_{\text {norm }}^{\alpha}\right\rangle$ components. Finally, in terms of the $\left|\tilde{\phi}^{\alpha}\right\rangle$, the effective Hamiltonian is given by $H_{e f f}=\sum_{\alpha}\left|\phi_{\text {norm }}^{\alpha}\right\rangle E^{\alpha}\left\langle\tilde{\phi}^{\alpha}\right|$ or $\left\langle i\left|H_{e f f}\right| j\right\rangle=$ $\sum_{\alpha}\left\langle i \mid \phi_{\text {norm }}^{\alpha}\right\rangle E^{\alpha}\left\langle\tilde{\phi}^{\alpha} \mid j\right\rangle$.

\section{Calculations}

In this work, we want to perform unrestricted shell-model calculations for the upper $p f$-shell nuclei ${ }^{64} \mathrm{Ge}$ and ${ }^{68} \mathrm{Se}$ using a recently developed effective interaction. We also would like to compare these results to the ones obtained some years ago with another effective interaction. The model spaces we work in are $p f_{5 / 2} g_{9 / 2}$ and its renormalized counterpart $p f_{5 / 2}$. Some typical dimensions that we deal with when performing $M$-scheme calculations in these model spaces are listed in Table 1 (where the number of $p f_{5 / 2} g_{9 / 2}$ basis state dimension put for the ${ }^{68} \mathrm{Se}$ isotope is for the case when time-reversal and charge-conjugation symmetries are employed by the code). The core nucleus for both choices is ${ }^{56} \mathrm{Ni}$, i.e. the $f_{7 / 2}$ orbital from the $p f$ 
Table 1. The dimensions of the model spaces $p f_{5 / 2} g_{9 / 2}$ and $p f_{5 / 2}$ used in our calculations (illustrated in terms of the number of basis states for the projection $M_{J}=0$ of the total angular momentum $J$ ) for the nuclei ${ }^{64} \mathrm{Ge}$ and ${ }^{68} \mathrm{Se}$.

\begin{tabular}{lll}
\hline nucleus & $p f_{5 / 2} g_{9 / 2}$ & $p f_{5 / 2}$ \\
\hline${ }^{64} \mathrm{Ge}$ & $1,831,531$ & 28,503 \\
${ }^{68} \mathrm{Se}$ & $1,929,014$ & 93,710 \\
\hline
\end{tabular}

shell is considered to be frozen while the $g_{9 / 2}$ orbital from the next $s d g$ shell is added to the model space.

The interaction that we used in the past for calculating these nuclei and to estimate the quality of their pseudo$S U(3)$ symmetry [8] is a result from a G-matrix calculation by Hjorth Jensen and Kuo with a later phenomenologically adjusted monopole part used in works like [9]. In the current work, we propose the use of a more recent one - the JUN45 effective interaction - introduced by Honma et al. [10]. Results on the pseudo-SU(3) symmetry study with the first interaction were reported in [8] using its renormalized version in the $p f_{5 / 2}$ model subspace (obtained with the projection method described in the previous paragraph).

In contrast with the previously used interaction, our current choice JUN45 has some extra features which favors its performance. For example, its two-body matrix elements depend on the mass number $A$ via the factor $A^{-0.3}$. This fact allows to better account of the properties of a specifically chosen nucleus (especially those not so close on the nuclear chart to the one for which these values give reasonable results).

In our calculations, we used the Glasgow Shell-model code [11]. Besides its benefits (it uses the so called $M$ scheme basis which is simple and easily worked with but does not have the total angular momentum $J$ as a good quantum number), it has some disadvantages one of which being its inability to handle too big model spaces (like the ones consisting of more than several million basis states). For that reason, in our future calculations we will most likely use shell-model codes that can handle bigger model spaces needed for such nuclei as the $M$-scheme shell model code ANTOINE [12].

Now, in order to estimate the goodness of some dynamical symmetry, we need to calculate the overlap between the energy eigenstates and the following two sets of eigenstates - of the quadrupole-quadrupole interaction (with rotational nature):

$$
\left|\Psi_{R}\right\rangle \equiv\left|\{\tilde{f}\} \alpha(\lambda, \mu) K L,\{f\} \beta(S T) M_{T} ; J M_{J}\right\rangle
$$

and of the total monopole pairing interaction (the isoscalar plus the isovector part used with equal strengths):

$$
\left|\Psi_{P}\right\rangle \equiv\left|\{\tilde{f}\} v\left[p_{1}, p_{2}, p_{3}\right] \xi L,\{f\} \beta(S T) M_{T} ; J M_{J}\right\rangle .
$$

The number of basis states from each of these types which are necessary to achieve a certain level of correspondence to the energy eigenfunctions can be our estimate of which between the two symmetries we study is present at a greater extent and can be considered to dominate. The two symmetries we study are the $S U$ (3) (or more correctly, the pseudo $S U(3)$ symmetry [13] since the calculations are in the $p f_{5 / 2}$ model space which is the model space for the upper $p f$-shell nuclei and the levels from the $p f$ shell can be relabelled as those in a shell of one quantum less than the one they originally belong while the broken symmetry in the initial configuration may be at least partially restored) and the $O(6)$ symmetry.

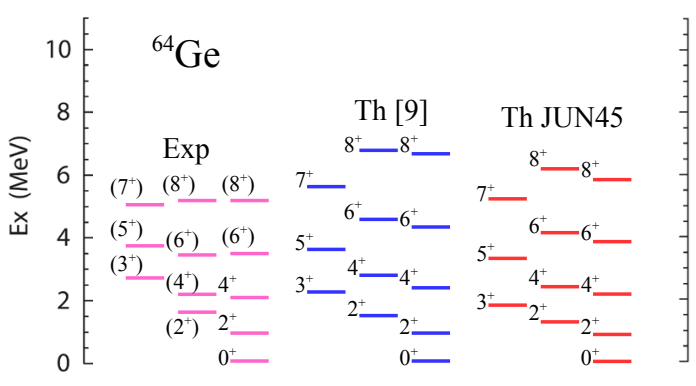

Figure 1. (Color online) Excitation energy spectrum of the g.s. band in ${ }^{64} \mathrm{Ge}$ obtained in the $p f_{5 / 2}$ model space using the JUN45 and the interaction from [9] compared with the experimental results [14].

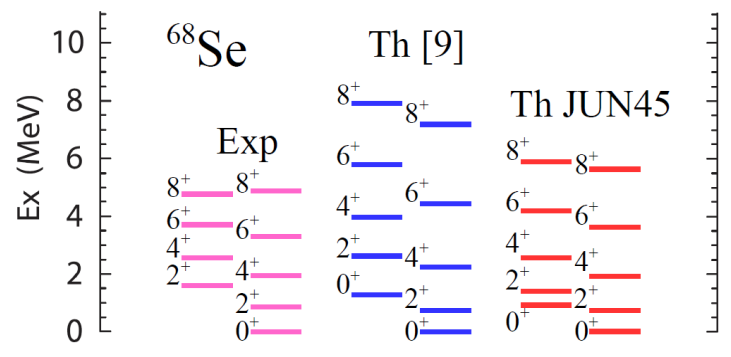

Figure 2. (Color online) Excitation energy spectrum of the g.s. band in ${ }^{68} \mathrm{Se}$ obtained in the $p f_{5 / 2}$ model space using the JUN45 and the interaction from [9] compared with the experimental results [14].

After we use the connection between the $S U(3)$ and the $M$-scheme basis states, we further apply the known approach developed in [15] for deriving a relation between the quadrupole-quadrupole and pairing states in the case of eight or twelve particles in the $s d$ shell. In order to speed up the calculations and to more clearly illustrate the $O(6)$ content in the energy eigenstates, only $S U(3)$ states and $O(6)$ components in the $S U(3)$ versus $O(6)$ expansion with the value for the amplitude of more than a 0.1 percent in the energy eigenstates have been included. Although this method is direct, its application is quite cumbersome and tedious. Alternatively, a straightforward application of the generalized seniority introduction [16] may be made for the total pairing chain of algebras $[S O(6) \supset S O(3)] \times U(4)$ and the connection between the pairing eigenstates in an orbit and the M-scheme basis can be established and used 
for finding an expression that relates the two types of bases acting in the whole oscillator shell.

Since the calculations for the nucleus ${ }^{68} \mathrm{Se}$ require to generate a huge matrix with dimensions on the order of several millions of $M$-scheme basis states (see Table 1), in this work we restricted ourselves to apply the projection method and to estimate the new matrix elements for the isotope ${ }^{64} \mathrm{Ge}$ only and then to use this result to both nuclei ${ }^{64} \mathrm{Ge}$ and ${ }^{68} \mathrm{Se}$. We expect that since these nuclei are quite close on the nuclear chart, the change between the matrix elements will not be that essential for the conclusions that will follow.

\section{Results: Excitation energy spectra and goodness of pseudo-SU(3) and O(6) symmetries}

The results obtained for the excitation energy spectra of the low-lying positive-parity states from the ground state and the excited state band with both effective interactions can be seen in Figs. 1 and 2 for the nuclei ${ }^{64} \mathrm{Ge}$ and ${ }^{68} \mathrm{Se}$, respectively. The outcome obtained is also compared to the experiment [14].

Clearly, JUN45 is the effective interaction which is more successful in the close description of the state positions and the overall characteristics of the spectra. As is clearly seen from the figures (especially in the case ${ }^{68} \mathrm{Se}$ ), JUN45 is the interaction that even for high $J$ values stays close to the experimental numbers. This result is partially due to the $A$-dependence of the JUN45 interaction mentioned above. Also, the obtained excitation energy ratio $\left.E_{x}\left(4_{1}^{+}\right) / E_{x}\left(2_{1}^{+}\right)\right)$values of $2.50(2.42)$ is closer to the experimental values of $2.28(2.27)$ for ${ }^{64} \mathrm{Ge}\left({ }^{68} \mathrm{Se}\right)$. For the isotope ${ }^{64} \mathrm{Ge}$, this result exactly matches the value corresponding to a gamma-soft nucleus with a good $O(6)$ symmetry.

In Fig. 3, one can see our results for the pseudo-SU(3) and the $O(6)$ symmetry quality in the ${ }^{64} \mathrm{Ge}$ and ${ }^{68} \mathrm{Se}$ isotopes, respectively. This is illustrated by showing the amplitudes with which $S U(3)(O(6))$ representations with a certain value of the second-order Casimir invariant of the $S U(3)$ algebra $C_{2}(S U(3))=1 / 4\left(Q . Q-3 \hat{L}^{2}\right)$ (the seniority $v)$ contribute to the energy eigenfunctions. Since the contribution to the eigenfunctions from the rotational states of low values of $C_{2}(S U(3))$ (and high values of the seniority number - beyond $v=4$ ) are typically small we either did not include them in the figures or we summed their cumulative effect.

From Fig. 3, parts (a) to (c), one can make a conclusion that for ${ }^{64} \mathrm{Ge}$ the goodness of the pseudo-SU(3) symmetry, although weakened compared to the earlier results with the interaction [9], seems to still be present and the first five $S U(3)$ irreducible representations account for upto about 80 percent of the energy eigenstates. The new result from the current work for this nucleus is that it can also be described upto a 95 percent by using the $O(6)$ pairing eigenstates of seniority values $v=0,2,4$ only. Beyond the $J=2(J=4)$ energy eigenstates, basis states of seniority $v=0(v=2)$ are not admissible according to their full classification but still the pairing basis states with upto $v=4$ are the only dominant $O(6)$ representations. These observations and the fact that the rest of the $O(6)$ total pairing eigenstates (of $v=6$ and 8) constitute the remaining majority (about 90 percent) of the model space leads us to the conclusion that this nucleus is also likely to be considered a gamma-soft rotor (as suggested by theoretical models and the experimental value for the excitation energy ratio). In short, both its energy excitation spectrum and the energy eigenstate structure point to that. The behavior of this isotope like an $S U(3)$-like nucleus (relatively strong $B E(2)$ transition strengths) stands against the inappropriate $E_{x}\left(J=4_{1}^{+}\right) / E_{x}\left(J=2_{1}^{+}\right)$ratio of 2.28 (not close to the value of 3.33 of the perfect rotator). Thus, the positive indications for the existence of $S U(3)$ symmetry should probably be attributed to the existence of a quasidynamical symmetry [17] (and more specifically - quasi $S U(3)$ ) which manifests itself in the coherent nature of the components of the energy eigenfunctions for the states between which electromagnetic transitions take place.

As for the ${ }^{68} \mathrm{Se}$ isotope (with results shown in Fig. 3, parts (d) and (e)), we conclude that it can not be so clearly claimed to be an isotope with good $O(6)$ symmetry since higher $v$ values (beyond $v=4$ and upto $v=12$ ) play a more significant role in building the eigenstates especially those from the excited state band. This nucleus may be more likely classified as a one of reasonable goodness of both pseudo-SU(3) and $O(6)$ symmetries without a clear preference to one of them. A further more detailed investigation on the interplay between these two symmetries would be quite an interesting topic.

Since we work in the upper $p f$-shell region of the nuclear chart (with a core the isotope ${ }^{56} \mathrm{Ni}$ ), the symmetry of the total monopole pairing interaction $S O(8)$ is naturally restricted to $S O(6)$ which follows from the fact that the spectrum generating shell-model algebra is $U(4 \times \Omega) \equiv$ $U(24)$ where the $s d$-shell spatial degeneracy is $\Omega=6$. Then, the spatial part of the chain in the classification of states follows as $U(\Omega) \supset S O(\Omega)$ and then the established complementarity $S O(\Omega) \sim S O(8)$ valid for any oscillator shell in the $s d$ shell becomes simply $S O(6)$. This symmetry is one of the dynamical symmetries in the Interacting Boson Model [1] so it is quite interesting to observe its existence (typical for the $s d$ shell nuclei) in some isotopes lying one shell higher, namely the $p f$ shell.

\section{Discussion and future work}

In our current work, we studied the symmetry properties of two $p f$-shell nuclei. We established that there are cases when there is more than one candidate with a reasonably good quality. It is quite interesting to study the interplay of these symmetries. One possible way to do that was suggested and extensively studied in [18] calling their study a mixed-mode or oblique basis approach. The interplay of the $S U(3)$ rotor and the spherical shell model single-particle states was reported. The results for the $s d$ shell isotope ${ }^{24} \mathrm{Mg}$ and the $p f$-shell isotope ${ }^{44} \mathrm{Ti}$ demonstrated several advantages achieved in this study over the 
(a)

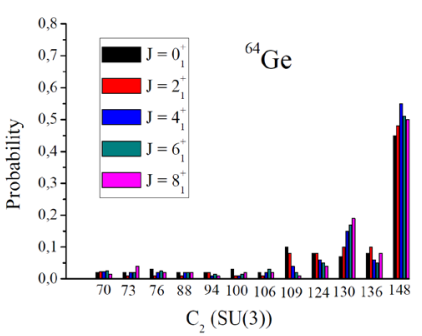

(b)

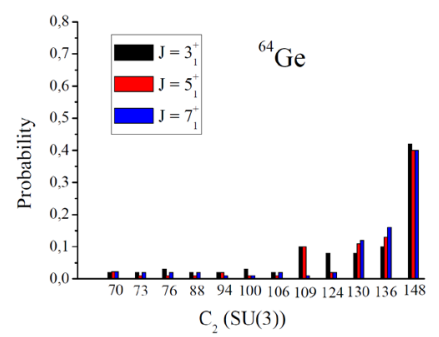

(c)

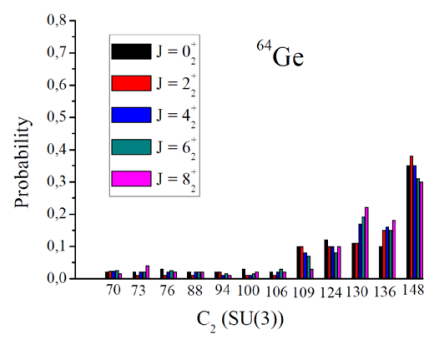

(d)

(e)
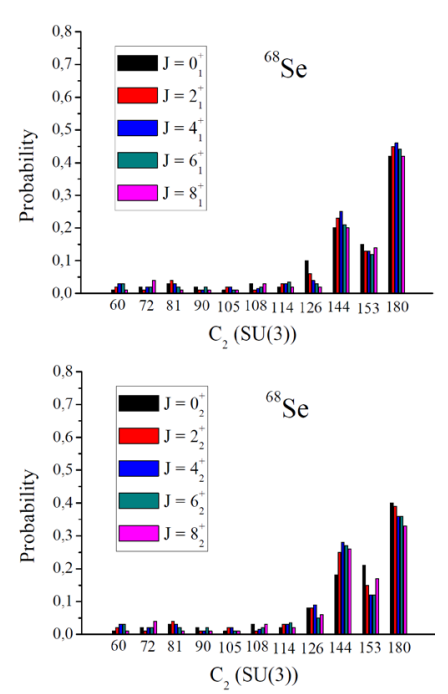
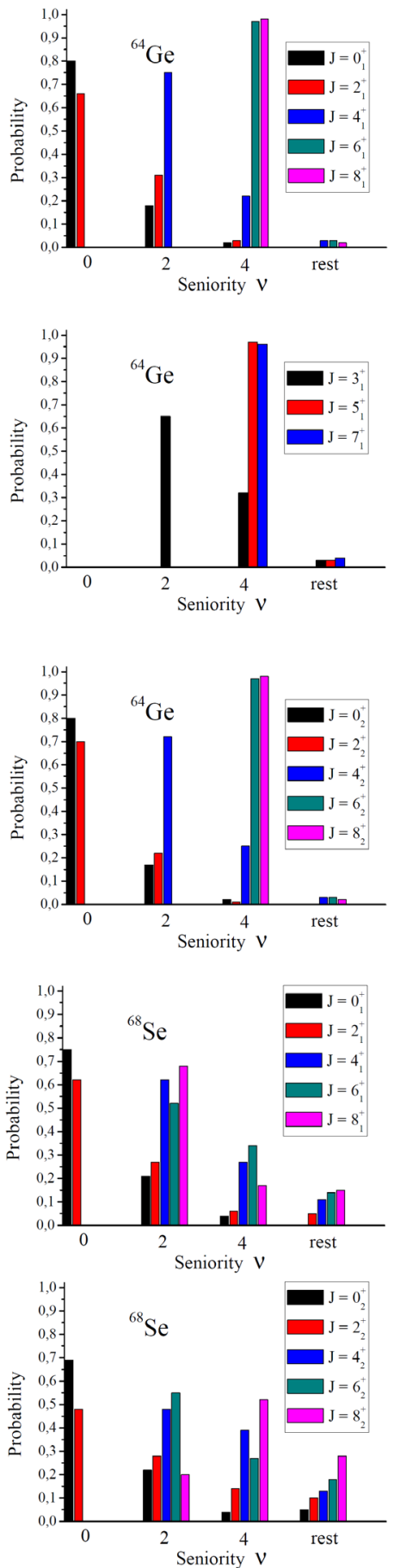

Figure 3. (Color online) Evaluation of the goodness of the pseudo-SU(3) and $O(6)$ symmetries in the positive-parity energy eigenstates from (a) the g.s. band and (b) and (c) the excited band of ${ }^{64} \mathrm{Ge}$. The same is given for ${ }^{68} \mathrm{Se}$ in parts (d) and (e) of the figure.

conventional single-mode basis approach. The work in a mixed basis takes both symmetries at a time and speeds up the rate of convergence of the energy eigenstates and the eigenfunctions towards the full-model space results. It was suggested that this approach can be continued as three- (or multiple-) mode calculation by putting together more than two modes expected to be important for a certain nucleus.
Our current study is a step towards including modes like the total monopole pairing in such kind of calculations. As past and current research activities, we explore the interplay of two, three and upto four independent interactions in $s d$-shell and $p f$-shell nuclei without any restrictions imposed on the single-shell model space. We study the interplay of the pairing (the total pairing inter- 
action with an overall strength or the isoscalar and isovector parts of it each with its independent strengths) and the quadrupole-quadrupole interaction [19] and even include the spin-orbit interaction as an additional term whose role we also evaluate [20]. It will be quite interesting (especially for the $p f$-shell nuclei) to study what restriction in the model will make sense for certain nuclei and which modes will appear to dominate in these results.

\section{Summary and conclusions}

To summarize, we demonstrated the quality of the symmetries pseudo-SU(3) and $O(6)$ in the upper $p f$-shell nuclei ${ }^{64} \mathrm{Ge}$ and ${ }^{68} \mathrm{Se}$. This was done by using the effective interaction JUN45 in the $p f_{5 / 2} g_{9 / 2}$ model space which was projected in the smaller $p f_{5 / 2}$ model space. Results for the goodness of the symmetries were compared with those obtained in the past using another effective interaction while the advantages over them were pointed out as well as the way some previous obstacles were overcome by using the current interaction. Finally, we commented on the possibility to make a generalization of a well-known approach for calculations in a mixed-symmetry basis to be applied in case of competing pairing and quadrupole modes (with the possibility of multi-mode further expansion). The goal of this future plan is that with its development we will accelerate the rate of convergence of our calculations compared to the use of only one of the bases. Thus, we may be able to also address the multi-shell calculations for these nuclei where the use of symmetries becomes crucial.

This work is supported by the Bulgarian National Foundation for Scientific Research under Grant Number DFNI-E02/6 (12.12.2014).

\section{References}

[1] A. Arima, F. Iachello, The Interacting Boson Model (Cambridge University Press, Cambridge, 2006)

[2] L. Wilets, M. Jean, Phys. Rev. 102, 788 (1956)

[3] A.S. Davydov, G.F. Filippov, Nucl. Phys. 8, 237 (1958)

[4] K. Kaneko, M. Hasegawa, T. Mizusaki, Phys. Rev. C 70, 051301 (2004)
[5] E. Caurier, G. Martinez-Pinedo, F. Nowacki, A. Poves, A.P. Zuker, Rev. Mod. Phys. 77, 427 (2005)

[6] B.R. Barrett, E.C. Halbert, J.B. McGrory, Ann. Phys. 90, 321 (1974)

[7] F. Monti, G. Bonsignori, M. Savoia et al., Nuov Cim A 104:33 (1991)

[8] K.P. Drumev, Louisiana State University, Ph.D. Dissertation (2008)

[9] P. Van Isacker, O. Juillet, F. Nowacki, Phys. Rev. Lett. 82, 2060 (1999)

[10] M. Honma, T. Otsuka, T. Mizusaki, and M. HjorthJensen, Phys. Rev. C 80, 064323 (2009)

[11] R.R. Whitehead, A. Watt, B.J. Cole, and I. Morrison, Adv. Nucl. Phys. 9, 123 (1977)

[12] E. Caurier, F. Nowacki, Acta Phys. Pol. B 30, 705 (1999)

[13] R.D. Ratna Raju, J.P. Draayer, K.T. Hecht, Nucl. Phys. A 202, 433 (1973)

[14] http://www.nndc.bnl.gov/

[15] J. Flores, E. Chacon, P.A. Mello, M. De Llano, Nucl. Phys. 72, 352 (1965); M. De Llano, P.A. Mello, E. Chacon, J. Flores, Nucl. Phys. 72, 379 (1965)

[16] A. Arima, H. Kawarada, J. Phys. Soc. Jpn 19, 1768 (1964)

[17] D.J. Rowe, Phys. Rev. Lett. 93, 122502 (2004); C. Bahri, D.J. Rowe, W. Wijesundera, Phys. Rev. C 58, 1539 (1998)

[18] V.G. Gueorguiev, Louisiana State University, Ph.D. Dissertation (2003); V.G. Gueorguiev, W.E. Ormand, C.W. Johnson, J.P. Draayer, Phys. Rev. C 65, 024314 (2002)

[19] K.P. Drumev, A.I. Georgieva, Nuclear Theory, Vol. 32 (2013) 151, Proc. 32 Int. Workshop on Nuclear Theory, 23 - 29 June 2013, eds. A. Georgieva, N. Minkov, Heron Press, Sofia. http://ntl.inrne.bas.bg/workshop/ 2013/contributions/a16_drumev.pdf

[20] K.P. Drumev, A.I. Georgieva, J. Cseh, Nuclear Theory, Vol. 35 (2016) 263, Proc. 35 Int. Workshop on Nuclear Theory, 26 June - 2 July 2016, eds. M. Gaidarov, N. Minkov, Heron Press, Sofia. 